\title{
EMISSÕES NATURAIS E ANTRÓPICAS DE NITROGÊNIO, FÓSFORO E METAIS PARA A BACIA DO RIO MACAÉ (MACAÉ, RJ, BRASIL) SOB INFLUÊNCIA DAS ATIVIDADES DE EXPLORAÇÃO DE PETROLEO E GÁS NA BACIA DE CAMPOS
}

\author{
Mauricio Mussi Molisani* e Franscisco de Assis Esteves \\ Núcleo em Ecologia e Desenvolvimento Sócio Ambiental de Macaé, Universidade Federal do Rio de Janeiro, Campus Macaé, Av. \\ São José do Barreto. 764, 27971-550 Macaé - RJ, Brasil \\ Luiz Drude de Lacerda \\ Instituto de Ciência do Mar, Universidade Federal do Ceará, Av. Abolição, 3207, 60165-081 Fortaleza - CE, Brasil \\ Carlos Eduardo de Rezende \\ Laboratório de Ciências Ambientais, Centro de Biociências e Biotecnologia, Universidade Estadual do Norte Fluminense, Av. \\ Alberto Lamego, 2000, 28013-602 Campos dos Goytacazes - RJ, Brasil
}

Recebido em 24/2/12; aceito em 14/8/12; publicado na web em 29/11/12

\begin{abstract}
NATURAL AND ANTHROPOGENIC EMISSIONS OF NITROGEN, PHOSPHOROUS AND METALS INTO THE MACAÉ RIVER BASIN (MACAÉ, RJ, BRAZIL) INFLUENCED BY OIL AND GAS EXPLORATION IN CAMPOS BASIN. Emission factors of natural processes and anthropogenic activities were used to estimate nutrients and metal loads for the lower Macaé river basin, which hosts the operational base for the offshore oil and gas exploration in the Campos Basin. The estimates indicated that emissions from anthropogenic activities are higher than natural emissions. Major contributing drivers include husbandry and urbanization, whose effluents receive no treatment. The increasing offshore oil exploration along the Brazilian littoral has resulted in rapid urbanization and, therefore might increase the inshore emission of anthropogenic chemicals in cases where effective residue control measures are not implemented in fluvial basins of the region.
\end{abstract}

Keywords: emission factor; pollutants; Macaé River.

\section{INTRODUÇÃO}

As emissões antrópicas de nutrientes $(\mathrm{N}, \mathrm{P})$ e metais de interesse ambiental ( $\mathrm{Zn}, \mathrm{Cu}, \mathrm{Pb}$ e $\mathrm{Cd})$ para muitos ecossistemas aquáticos vêm superando de forma quantitativa as emissões naturais, oriundas da lixiviação de solos e da deposição atmosférica, e alterando sua composição, induzindo a eutrofização e a contaminação da biota. ${ }^{1-3}$ Este aumento está principalmente relacionado ao crescimento populacional e à demanda por bens de consumo e serviços que geram resíduos contendo nutrientes e metais, devido à disposição inadequada de rejeitos sólidos, ${ }^{4-6}$ lançamento de esgoto doméstico sem tratamento, escoamento superficial (runoff) urbano, ${ }^{7-9}$ liberação de resíduos agrícolas de fertilizantes e biocidas ${ }^{10-14}$ ou por meio de fezes e urina de animais oriundos de atividades pecuárias. ${ }^{15}$

A exploração de petróleo e gás em bacias petrolíferas marinhas é uma atividade econômica crescente na costa brasileira e pode gerar emissões de substâncias que são usualmente relacionadas a derrames de petróleo e outros efluentes relativos à produção em águas costeiras e oceânicas. ${ }^{16,17}$ Por outro lado, a exploração offshore de petróleo e gás como, por exemplo, na Bacia de Campos (RJ) demanda os mais diversos serviços que necessitam de uma crescente população e da diversificação das atividades econômicas localizadas no continente que podem induzir modificações em ecossistemas continentais distantes das áreas de produção em alto mar. Como exemplo, pode-se relatar o crescimento populacional e econômico em regiões como o município de Macaé (RJ), que é a base operacional das atividades de exploração de petróleo e gás na Bacia de Campos, que pode estar induzindo o aumento das emissões de nutrientes e metais para os ecossistemas da região, gerando pressão sobre os recursos naturais.

*e-mail: molisanimm@yahoo.com.br
A bacia hidrográfica do Rio Macaé é um conjunto de ecossistemas que promovem importantes serviços econômicos e ambientais para as atividades de exploração de petróleo e gás na Bacia de Campos, embora estejam ocorrendo modificações importantes neste ecossistema aquático desde a instalação da estrutura para exploração na década de 70, que desencadeou um acentuado crescimento econômico no município de Macaé e região. A expansão da produção em novos poços na Bacia de Campos tem atraído novos investimentos e levado ao aumento exponencial da indústria de serviços. O reflexo do aumento populacional associado à ocupação desordenada e à falta de tratamento dos resíduos gerados como, por exemplo, a ausência do tratamento de esgoto e seu lançamento in natura são responsáveis pela poluição hídrica desta região. Além das atividades de serviços vinculadas ao setor petrolífero, destacam-se algumas atividades econômicas como agricultura, pecuária, geração de energia e turismo que podem incrementar as emissões naturais de nutrientes e metais para o Rio Macaé e que também vêm sendo incrementadas pela atividade petrolífera.

Mesmo considerando o crescimento econômico para a região impulsionado pela economia do petróleo e o aumento nas emissões de nutrientes e metais para seus ecossistemas, observa-se a ausência de estudos sistemáticos sobre as concentrações e os fluxos de espécies químicas para as diversas bacias de drenagem da região, principalmente em bacias hidrográficas de pequeno e médio porte, como o Rio Macaé. Na ausência de dados primários, o uso de fatores de emissão de nutrientes e metais pode ser uma ferramenta para quantificar a importância de fontes naturais e antrópicas para bacias fluviais, quando não há informações sistemáticas sobre concentrações e fluxos visando avaliar de forma preliminar a sensibilidade de ecossistemas aquáticos ao aporte antrópico adicional de substâncias de interesse ambiental. Assim, o presente estudo visou estimar, através do cálculo dos fatores de emissão, a carga anual de $\mathrm{N}, \mathrm{P}, \mathrm{Zn}, \mathrm{Cu}, \mathrm{Pb}$ e 
$\mathrm{Cd}$ oriundos de fontes naturais e antrópicas para a bacia hidrográfica do Rio Macaé, que recentemente vem sofrendo alterações devido ao crescimento populacional e econômico induzido pelas atividades de exploração de petróleo e gás na Bacia de Campos.

\section{PARTE EXPERIMENTAL}

\section{Área de estudo}

A bacia do Rio Macaé possui uma área de drenagem de $1.765 \mathrm{~km}^{2}$ ao longo da região serrana e norte fluminense do Estado do Rio de Janeiro, compreendendo seis municípios, embora $82 \%$ da área da bacia estejam no município de Macaé. O curso do rio é de $136 \mathrm{~km}$, sendo que na porção superior o rio nasce em relevo com altitude máxima de $1560 \mathrm{~m}$, enquanto que na bacia inferior o rio passa através de uma planície aluvial antes de desaguar no Oceano Atlântico, através do seu estuário localizado na zona urbana do município de Macaé. A bacia hidrográfica tem uso do solo determinado por fragmentos florestais (41\%), pasto (34\%), áreas agrícolas (19\%), urbanização (3\%) e outros $3 \%$ com várias tipologias, tais como áreas inundáveis, corpos d'água, solo exposto, coberturas de manguezais. ${ }^{18}$ A partir da década de 70 quando começaram as atividades de exploração de petróleo e gás na Bacia de Campos, a população de Macaé cresceu 4,4 vezes, de 47.220 em 1970 para 206.728 em 2011. ${ }^{19}$ Adicionalmente pode-se observar uma taxa anual de crescimento econômico de aproximadamente $6 \%$ nos últimos 10 anos, que é superior ao crescimento médio $(0,5 \%)$ estimado para o estado do Rio de Janeiro nesse mesmo período. Este crescimento em um curto período de tempo potencializou um aumento na emissão de nutrientes e metais para a bacia do Rio Macaé com efeitos ainda não determinados no Rio Macaé.

\section{Estimativa dos fatores de emissão}

Os fatores de emissão (FEs) são largamente utilizados para gerar inventários de cargas de espécies químicas para o ambiente quando da inexistência de medições reais. Os FEs são geralmente expressos em unidade de massa por período de tempo relativo a processos naturais e de atividades antrópicas, calculados a partir de parâmetros de produção e/ou consumo de fontes antrópicas e do balanço químico de processos naturais. ${ }^{20}$ FEs vêm sendo utilizados com aceitável exatidão como metodologia padrão para estimar as emissões a nível local, ${ }^{3,9,21}$ regional $^{1,2,22-25}$ e global ${ }^{20,26,27}$ por diversas agências ambientais. ${ }^{28}$ Em geral, os fatores de emissão utilizados neste estudo são calculados a partir de parâmetros locais disponibilizados na literatura e, na ausência destas informações, são utilizados fatores gerais de emissões globais. Considerando que $82 \%$ da área da bacia do Rio Macaé compreende o município de Macaé, as cargas anuais foram calculadas para as bacias média e inferior, a partir dos dados relativos ao município. Os FEs de fontes antrópicas levam em consideração relações entre as atividades produtivas como dados de produção, uso de insumos, bem como as concentrações das espécies químicas nas diversas matrizes analisadas e em seus efluentes, incluindo variáveis como as taxas de retenção em solos, por exemplo. ${ }^{21,29-36}$ Os FEs de fontes naturais de N, P e metais, como a lixiviação de solos levaram em consideração a composição e distribuição dos solos na região, as taxas de perda de solo sob diferentes usos e taxas de retenção ou liberação das espécies químicas de interesse. Para as estimativas naturais oriundas da deposição atmosférica foram utilizadas taxas de deposição atmosférica e respectivas concentrações das substâncias de interesse de áreas com características ambientais semelhantes. ${ }^{6,9,37-40}$ As equações utilizadas e memórias de cálculo para cada fonte de emissão e espécies químicas avaliadas estão detalhadas nas tabelas nas quais os resultados são apresentados.
A avaliação da exatidão dos fatores de emissão foi feita por determinação e/ou medição dos fluxos de $\mathrm{N}$ e P e dos metais na porção final da bacia do Rio Macaé considerando que todas as emissões irão atingir esta região antes do rio desaguar no Oceano Atlântico. Os fluxos foram calculados considerando a relação entre a vazão medida por um correntômetro de efeitos Doppler (ADP) e as concentrações totais de espécies químicas determinadas em amostras de água da superfície e fundo da coluna d'água, coletadas em três pontos da seção transversal do rio. As concentrações de $\mathrm{N}$ e $\mathrm{P}$ total foram determinadas por espectrofotometria UV-Visível utilizando-se persulfato de potássio como extrator. ${ }^{41}$ Os metais foram determinados no material particulado em suspensão, que usualmente é o principal suporte geoquímico destes elementos, responsável pela maior parte do transporte de metais no ambiente fluvial. A concentração dos metais na fase particulada foi obtida através de filtração por membranas Whatman GF/F e extração ácida, sendo os extratos analisados por espectrometria de emissão óptica por plasma acoplado indutivamente - ICP OES. ${ }^{42}$

\section{RESULTADOS E DISCUSSÃO}

\section{Emissões naturais: lixiviação de solos e deposição atmosférica}

Os solos são importantes compartimentos reservatórios de elementos químicos oriundos da rocha matriz e da deposição atmosférica, embora as ações de processos erosivos de natureza física, química e biológica atuem para efetivar os solos como fontes de $\mathrm{N}, \mathrm{P}, \mathrm{Zn}, \mathrm{Cd}, \mathrm{Cu}$ e $\mathrm{Pb}$ para ambientes fluviais. Estes processos são influenciados pelo tipo de solo e pelos aspectos climáticos da região. Por outro lado, as perdas de solo são aceleradas pelas diferentes práticas agrícolas, assim como pela urbanização, podendo alcançar 116 a 309 t km$^{-2}$ ano $^{-1}$ em regiões de clima temperado ${ }^{43}$ e 60 a 760 $\mathrm{t} \mathrm{km}^{-2}$ ano $^{-1}$ para regiões tropicais (média de $128 \mathrm{t} \mathrm{km}^{-2} \mathrm{ano}^{-1}$ ). ${ }^{44} \mathrm{O}$ valor médio de $130 \mathrm{t} \mathrm{km}^{-2}$ ano $^{-1}$ foi reportado para áreas de baixa inclinação, sem utilização de agricultura mecanizada na região africana tropical. ${ }^{45}$ Considerando a variabilidade das taxas de perdas de solo para regiões tropicais, muitas das quais com características similares à bacia do Rio Macaé, foi utilizado o valor de $128 \mathrm{t} \mathrm{km}^{-2}$ $\mathrm{ano}^{-1}$ para os cálculos das emissões de nutrientes e metais pela perda de solo e as faixas de concentrações reportadas para os diferentes tipos de solo encontrados na região sudeste. Estas concentrações variaram de acordo com o tipo de solo para $\mathrm{N}$ : 500-900 $\mathrm{mg} \mathrm{g}^{-1} ; \mathrm{P}$ : 100-500 $\mathrm{mg} \mathrm{g}^{-1}$; Zn: 21-126 g g $^{-1}$; Cu: 9 -26 $\mu \mathrm{g} \mathrm{g}^{-1}$; Pb: 0,8-53 $\mu \mathrm{g}$ $\mathrm{g}^{-1}$ e Cd: $0,1-2,0 \mu \mathrm{g} \mathrm{g}^{-1} \cdot$. $^{13,14,46-54}$

Os principais tipos de solos da área de estudo são latossolos, cambissolos, podsólicos, areias e gleissolos, ${ }^{55}$ totalizando uma área de $1092 \mathrm{~km}^{2}$ (Tabela 1). O restante da área, incluindo corpos d'água, rocha exposta e mangue, não foi considerado no inventário de emissões naturais por lixiviação de solos. A Tabela 1 mostra as emissões calculadas de N, P, Zn, Cd, Cu e Pb para cada tipo de solo, expressas em t ano $^{-1}$. Quando estes dados são avaliados considerando as perdas por unidade área pode-se observar uma maior contribuição dos cambissolos para N, P, Zn, Pb (204/3,84/8,7/3,8 kg km² ano, respectivamente) e podsólicos para $\mathrm{Cu}\left(2,3 \mathrm{~kg} \mathrm{~km}^{2}\right.$ ano).

Do mesmo modo, a deposição atmosférica é uma importante fonte de emissão natural de nutrientes e metais para bacias hidrográficas, sendo determinada pela área da bacia, a taxa de pluviosidade anual e a concentração dos elementos químicos da deposição total (seca e úmida). A deposição atmosférica total vem sendo medida para diversas áreas da costa brasileira, sendo que para elementos não quantificados neste estudo são listados valores típicos de outras áreas do mundo pouco ou não afetadas por atividades antrópicas, como a região norte fluminense. ${ }^{6,37-40,56-61}$ Adicionalmente, a fração dos elementos oriundos da deposição atmosférica que é transferida 
Tabela 1. Emissões de N, P, Zn, Cd, Cu e Pb (t ano $\left.{ }^{-1}\right)$ oriundos da lixiviação e perda dos diferentes tipos de solos da bacia média e inferior do Rio Macaéa

\begin{tabular}{lccccccc}
\hline Tipos de solo & Área $\left(\mathrm{km}^{2}\right)$ & $\mathrm{N}$ & $\mathrm{P}$ & $\mathrm{Zn}$ & $\mathrm{Cu}$ & $\mathrm{Pb}$ \\
\hline Latossolos & 162 & 12 & 0,15 & 0,36 & 0,25 & 0,35 & 0,029 \\
Cambissolos & 507 & 103 & 1,9 & 4,40 & 0,65 & 1,9 & 0,071 \\
Podsolos (Argissolos) & 104 & 8,0 & 0,13 & 0,72 & 0,24 & 0,27 & 0,008 \\
Areias (Espodolssolos, Neossolos) & 252 & 4,4 & 0,18 & 0,56 & 0,38 & 0,55 \\
Gleissolos (Aluviais/Regossolos) & 67 & 5,2 & 0,06 & 0,15 & 0,10 & 0,15 \\
\hline Total & 1092 & 124 & 2,23 & 5,50 & 1,14 & 0,045 & 2,57 \\
\hline
\end{tabular}

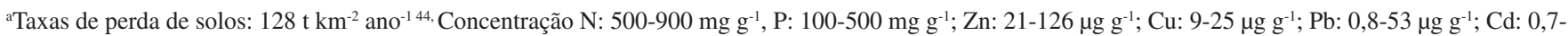
$2,0 \mu \mathrm{g} \mathrm{g}^{-1}$; em solos de áreas litorâneas de baixo desenvolvimento industrial ${ }^{13,14,46-54}$. ${ }^{6}$ Tipos de solo da bacia do Rio Macaé ${ }^{55}$

ao longo da bacia hidrográfica irá depender da taxa de retenção pelos diferentes tipos de solo. ${ }^{27,29,57}$

As emissões dos elementos para a bacia do Rio Macaé oriundas da deposição atmosférica foram quantificadas (Tabela 2). Foi considerada a pluviosidade média anual de $1.330 \mathrm{~mm}$ para a área de estudo. A deposição atmosférica foi estimada a partir das concentrações dos elementos típicas de áreas com baixa taxa de urbanização e industrialização, e das taxas de retenção de cada elemento pelos solos típicos da bacia. Comparando as cargas por fontes naturais observa-se que para elementos como $\mathrm{N}$ e $\mathrm{Pb}$ a lixiviação dos solos é a principal fonte natural para o Rio Macaé (81 e 70\%, respectivamente), enquanto que para $\mathrm{Cd}$ a deposição atmosférica tem maior participação na carga total (93\%). Para elementos como $\mathrm{P}, \mathrm{Zn}$ e Cu ambas as fontes naturais têm participação similar. As emissões naturais oriundas da lixiviação de solos e deposição atmosférica para o Rio Macaé estão dentro das faixas reportadas para outras bacias costeiras brasileiras com baixo desenvolvimento urbano/industrial..$^{2,21}$

\section{Emissões antrópicas: agricultura e pecuária}

As atividades humanas estão baseadas na utilização de recursos naturais e na emissão de resíduos para o ambiente, tornando-se, portanto, fontes adicionais de nutrientes e metais em relação às fontes naturais oriundas da denudação e lixiviação de solos e da deposição atmosférica. Dentre as atividades antrópicas, a agricultura e a pecuária podem ser fontes representativas na emissão dos elementos químicos estudados para a bacia do Rio Macaé.

As culturas agrícolas permanentes e temporárias ocupam uma área de 2892 ha na área compreendida da bacia do Rio Macaé, segundo o censo de $2010 .{ }^{19}$ As estimativas de emissão dos elementos por estas culturas consideram o uso de fertilizantes e defensivos agrícolas tendo em suas composições N, P e metais que são lixiviados para as águas superficiais dependendo do tipo do solo e da prática agrícola. Para estas culturas podem-se verificar na literatura aplicações de fertilizantes com teores de $\mathrm{N}$ e $\mathrm{P}$ variando de 20 a 275 e 12 a $70 \mathrm{~kg}$ $\mathrm{ha}^{-1}$, respectivamente..$^{29-31,62-65}$ Dependendo do tipo do solo podem-se relatar perdas de nutrientes oriundas de fertilizantes variando de 16 a $32 \%$ para $\mathrm{N}$ e 0,7 a $25 \%$ para $\mathrm{P}$. Solos agrícolas podem perder até $200 \mathrm{~kg} \mathrm{P} \mathrm{km}^{-2}{ }^{29,65,66}$ Do mesmo modo, insumos como fungicidas e fertilizantes, na forma de superfosfatos e ureia possuem concentrações mensuráveis de metais associados, originados na própria matriz geológica dos fosfatos e como impurezas residuais dos processos produtivos da ureia. Essas concentrações tendem a variar de acordo com a origem da matéria-prima utilizada na produção destes insumos. As concentrações de metais nos insumos tipicamente utilizados na agricultura têm sido bastante estudadas, sendo possível obter concentrações médias de resultados publicados na literatura. Estas concentrações foram utilizadas para calcular as cargas de $\mathrm{Cd}, \mathrm{Zn}, \mathrm{Cu}$ e $\mathrm{Pb}$ geradas para tipo de cultivo..$^{10-14}$

Do mesmo modo, a pecuária pode ser uma importante fonte de emissão de nutrientes considerando o tipo e tamanho do rebanho, as taxas de excreção dos animais e as concentrações dos elementos na fonte nutricional. Alguns trabalhos mostram taxas de excreção variando entre 0,2 a $10 \mathrm{~kg}$ animal ${ }^{-1}$ dia $^{-1}$ dependendo da criação, com concentrações de $\mathrm{N}$ e $\mathrm{P}$ variando entre 0,5 a 1,2\% e 0,3-1,3\%, respectivamente. ${ }^{67-69}$ Uma vez transferidos ao solo, as emissões de elementos oriundas das atividades pecuárias vão estar sujeitas às taxas de retenção de solos, como exemplificado na Tabela 2, antes de atingirem as águas superficiais. Segundo estimativas, 20-35\% do $\mathrm{N}$ e $35-60 \%$ do $\mathrm{P}$ presentes nas fezes dos animais vão alcançar as águas superficiais. ${ }^{28,64-70}$

Segundo as estimativas de emissões por atividades agropecuárias, pode-se observar que a pecuária é a principal fonte emissora de nutrientes e metais analisados para a bacia do Rio Macaé considerando a área cultivável da bacia que é expressiva somente para a cultura de banana, em comparação com a criação de gado de corte que é vocação regional (Tabela 3).

\section{Emissões antrópicas: escoamento superficial (runoff) urbano, resíduos sólidos e águas servidas (esgoto doméstico)}

Além das fontes oriundas da agropecuária, o crescimento populacional e de atividades econômicas na bacia do Rio Macaé, fomentados pelas atividades de exploração de petróleo na Bacia de

Tabela 2. Contribuição das emissões naturais de $\mathrm{N}, \mathrm{P}, \mathrm{Zn}, \mathrm{Cd}, \mathrm{Cu}$ e $\mathrm{Pb}\left(\mathrm{t}\right.$ ano $\left.{ }^{-1}\right)$ oriundas de deposição atmosférica e lixiviação e perda dos solos para a porção média e inferior do Rio Macaé

\begin{tabular}{lcccccc}
\hline & $\mathrm{N}$ & $\mathrm{P}$ & $\mathrm{Zn}$ & $\mathrm{Cu}$ & $\mathrm{Pb}$ & $\mathrm{Cd}$ \\
\hline Deposição atmosférica $^{\mathrm{a}}$ & 30 & 2,0 & 5,4 & 0,80 & 1,5 & 1,5 \\
Runoff de solos $^{\mathrm{b}}$ & 124 & 2,2 & 5,5 & 1,1 & 2,6 & 0,11 \\
Emissão total por fontes naturais & 153 & 4,2 & 11 & 1,9 & 3,7 & 1,60 \\
\hline
\end{tabular}

${ }^{a}$ Estimado pela média de deposição atmosférica relatada para áreas litorâneas de baixo desenvolvimento industrial (N: 50-640 $\mathrm{mg} \mathrm{m}^{-2}$ ano-1 $; \mathrm{P}^{-} 0,5-10 \mathrm{mg} \mathrm{m}^{-2}$ ano $^{-1}$; $\mathrm{Cu}$ 0,28-3,5 $\mathrm{mg} \mathrm{m}^{-2}$ ano $^{-1}$; Zn: 0,9-9,3 $\mathrm{mg} \mathrm{m}^{-2}$ ano $^{-1}$; Pb: 2,7-15 $\mathrm{mg} \mathrm{m}^{-2}$ ano $^{-1}$; Cd: 0,4-5,0 $\mathrm{mg} \mathrm{m}^{-2}$ ano $\left.^{-1}\right)^{6,37-40,54,56-61}$ ponderado pela pluviosidade média anual da bacia $(1330 \mathrm{~mm})$ e corrigido pelo fator de retenção de cada elemento no solo (N: 0,37; P: 0,30; Cu, Pb: 0,35; Zn: 0,$45 ; \mathrm{Cd}: 0,40)^{27,29,57}$ 
Tabela 3. Estimativa das emissões de $\mathrm{N}, \mathrm{P}, \mathrm{Zn}, \mathrm{Cd}, \mathrm{Cu}$ e $\mathrm{Pb}\left(\mathrm{t}\right.$ ano $\left.{ }^{-1}\right)$ para a bacia do Rio Macaé oriundas de fontes antrópicas

\begin{tabular}{|c|c|c|c|c|c|c|}
\hline & $\mathrm{N}$ & $\mathrm{P}$ & $\mathrm{Zn}$ & $\mathrm{Cu}$ & $\mathrm{Pb}$ & $\mathrm{Cd}$ \\
\hline Agricultura $^{\mathrm{a}}$ & 17 & 4,1 & 0,023 & 0,28 & $<0,001$ & 0,001 \\
\hline Pecuáriab & 754 & 459 & 7,400 & 1,40 & 0,110 & 0,005 \\
\hline
\end{tabular}

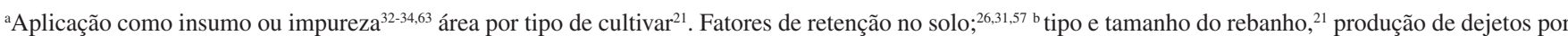
$\operatorname{animal}^{68}\left(\mathrm{~kg}\right.$ cabeça $^{-1}$ dia $\left.^{-1}\right)$, concentração dos elementos em dejetos ${ }^{12,69}$

Campos, induzem ao aumento da importância de fontes antrópicas relacionadas à urbanização como a geração e deposição inadequada de resíduos sólidos, de águas servidas (esgotos domésticos) e escoamento urbano. Via de regra estes efluentes não sofrem nenhum tipo de tratamento prévio ao seu lançamento no ambiente.

Os esgotos domésticos são importantes fontes de nutrientes e metais para as águas superficiais e são diretamente proporcionais ao número de habitantes, consumo de água por habitante, além das concentrações destes elementos químicos nos efluentes, que geralmente que possuem reduzida variabilidade..$^{8,36,65,71-73} \mathrm{~A}$ partir da década de 70 a população do município de Macaé aumentou de 47.220 para os atuais 206.218 habitantes, ${ }^{19}$ induzindo um aumento no consumo de água que, segundo estimativas por habitante, pode chegar a $220 \mathrm{~L} \mathrm{dia}^{-1}$ para população urbana e $125 \mathrm{~L} \mathrm{dia}^{-1}$ para população rural. ${ }^{74}$ Esta água utilizada ou servida retorna à bacia do Rio Macaé com a qualidade depreciada, visto que não há tratamento de esgotos ao longo da bacia. Do mesmo modo que a emissão por águas servidas, a emissão de elementos químicos pela geração de resíduos sólidos e pela lixiviação e escoamento pelo assoalho urbano estão diretamente associados ao grau de urbanização e podem contribuir para o enriquecimento destes elementos na água superficiais. Os principais parâmetros controladores destas emissões são o tamanho da população, as taxas de produção de resíduos e as concentrações dos elementos e formas de disposição dos rejeitos sólidos, além da área da superfície urbana impermeável e a pluviosidade. A falta de fatores de emissão para $\mathrm{P}$ oriundo da disposição de rejeitos sólidos impede os cálculos para este elemento.

A Tabela 4 apresenta a estimativa de cargas de nutrientes e metais pelos diversos vetores associados à urbanização. A carga originada na liberação de águas servidas é a principal fonte de $\mathrm{N}, \mathrm{P}, \mathrm{Zn}, \mathrm{Cu}$ e $\mathrm{Cd}$ para a bacia do Rio Macaé, enquanto que para $\mathrm{Pb}$ a disposição inadequada de rejeitos sólidos urbanos é a principal origem da carga. As emissões pelo escorrimento superficial urbano são relativamente pequenas quando comparadas àquelas dos dois outros vetores.

A análise integrada das emissões de nutrientes e metais oriundas de fontes naturais e antrópicas indica que atualmente as fontes antrópicas correspondem à quase totalidade da emissão de $\mathrm{N}$ e $\mathrm{P}$ e pela maior parte do $\mathrm{Zn}, \mathrm{Cu}, \mathrm{Pb}$ e $\mathrm{Cd}$ que atingem a bacia do Rio Macaé e podem ser eventualmente transferidas para a zona costeira (Tabela 5).

Segundo estas estimativas, as fontes antrópicas como a pecuária, as águas servidas e a disposição de resíduos sólidos são responsáveis pela maior parte destas emissões para o Rio Macaé ( $\mathrm{P}=89 \%$, $\mathrm{N}=99 \%, \mathrm{Zn}=62 \%, \mathrm{Cu}=65 \%, \mathrm{Cd}=60 \%$ ), exceto para $\mathrm{Pb}$ onde a deposição atmosférica e o escoamento superficial representam aproximadamente $55 \%$ do total (Tabela 5). Dentre estas atividades, as gerações de esgoto e resíduos sólidos estão relacionadas com o crescimento populacional e a urbanização, que vêm sendo estimuladas pelas atividades de exploração de petróleo na Bacia de Campos não somente na bacia do Rio Macaé, mas em outras bacias hidrográficas da região sob influência destas atividades.

A influência antrópica na emissão de elementos químicos é descrita para várias bacias fluviais do Brasil, estando relacionada às atividades socioeconômicas específicas de cada região. ${ }^{2,21,22,75}$ Comparadas com bacias com baixo índice de urbanização e tipicamente agrícolas, as emissões normalizadas $\left(\mathrm{kg} \mathrm{km}^{-2} \mathrm{ano}^{-1}\right)$ da bacia do Rio Macaé são superiores, refletindo a influência da recente urbanização da região. Porém, as emissões de N e P são inferiores a bacias altamente urbanizadas ou sob influência intensiva de atividades como a carcinicultura (Tabela 6).

\section{Incertezas das estimativas dos fatores de emissão}

As incertezas dos fatores de emissão refletem a falta de conhecimento sobre a real quantidade emitida por uma categoria de fonte $\mathrm{e}$ estão relacionadas à disponibilidade e variabilidade de populações de dados, que podem hierarquizar diversos fatores de emissão quanto à representatividade e utilização dos resultados. ${ }^{76}$ Os fatores de emissão são usualmente expressos na forma de valores médios ${ }^{2,3,21,22,24,27,34} \mathrm{ou}$ através da faixa de variação das emissões. ${ }^{21,23}$ Para estudos onde há obtenção de dados sistemática e abrangente, as incertezas podem ser

Tabela 4. Estimativa das emissões de $\mathrm{N}, \mathrm{P}, \mathrm{Zn}, \mathrm{Cd}, \mathrm{Cu}$ e $\mathrm{Pb}(\mathrm{t}$ ano-1 $)$ para a bacia do Rio Macaé oriundas de fontes antrópicas

\begin{tabular}{lcccccc}
\hline & $\mathrm{N}$ & $\mathrm{P}$ & $\mathrm{Zn}$ & $\mathrm{Cu}$ & $\mathrm{Pb}$ & $\mathrm{Cd}$ \\
\hline Águas servidas $^{\mathrm{a}}$ & 669 & 319 & 5,50 & 1,90 & 0,80 & 0,11 \\
Rejeitos sólidos $^{\mathrm{b}}$ & 0,31 & - & 5,10 & 1,50 & 3,20 & 0,32 \\
Escoamento urbano $^{\mathrm{c}}$ & 6,3 & 0,94 & 0,05 & 0,01 & 0,02 & 0,65 \\
\hline
\end{tabular}

${ }^{\mathrm{a}}$ Concentração em águas servidas, ${ }^{65,71-73}$ consumo/emissão de água servida, ${ }^{74}$ número de habitantes. ${ }^{19}$ b Concentração dos elementos em rejeitos sólidos urbanos, ${ }^{20,74}$ produção de rejeitos por habitante, ${ }^{4,6,35}$ número de habitantes dentro da bacia do Rio Macaé. ${ }^{19}$ cPluviosidade anual, ${ }^{3}$ concentração de elementos no runoff urbano ${ }^{7-9}$

Tabela 5. Comparação entre as cargas naturais e antrópicas $\left(\mathrm{t}\right.$ ano $\left.{ }^{-1}\right)$ de $\mathrm{N}, \mathrm{P}, \mathrm{Zn}, \mathrm{Cd}, \mathrm{Cu}$ e $\mathrm{Pb}$ para a bacia média e inferior do Rio Macaé

\begin{tabular}{lcccccc}
\hline & $\mathrm{N}$ & $\mathrm{P}$ & $\mathrm{Zn}$ & $\mathrm{Cu}$ & $\mathrm{Pb}$ & $\mathrm{Cd}$ \\
\hline Emissões naturais & 153 & 4,2 & 11 & 1,9 & 3,7 & 1,6 \\
Emissões antrópicas & 1446 & 783 & 18 & 5,1 & 4,2 & 2,1 \\
Emissão total para a bacia & 1599 & 787 & 29 & 7,0 & 7,9 & 3,7 \\
\% contribuição antrópica & 90 & 99 & 62 & 73 & 53 & 57 \\
\hline
\end{tabular}


Tabela 6. Fatores de emissão expressos por unidade de área $\left(\mathrm{kg} \mathrm{km}^{-2} \mathrm{ano}^{-1}\right)$ de $\mathrm{N}, \mathrm{P}, \mathrm{Zn}, \mathrm{Cd}, \mathrm{Cu}$ e $\mathrm{Pb}$ originados de processos naturais e atividades antrópicas na bacia média e inferior do Rio Macaé

\begin{tabular}{|c|c|c|c|c|c|c|}
\hline & $\mathrm{N}$ & $\mathrm{P}$ & $\mathrm{Zn}$ & $\mathrm{Cu}$ & $\mathrm{Pb}$ & $\mathrm{Cd}$ \\
\hline Deposição atmosférica & 24 & 1,8 & 4,5 & 0,90 & 2,1 & 0,09 \\
\hline Runoff de solos & 102 & 1,8 & 4,5 & 0,90 & 2,1 & 0,09 \\
\hline Agricultura & 14 & 3,4 & 0,02 & 0,23 & 0,001 & 0,001 \\
\hline Pecuária & 620 & 377 & 6,1 & 1,2 & 0,09 & 0,004 \\
\hline Águas servidas & 550 & 262 & 4,5 & 1,5 & 0,66 & 0,88 \\
\hline Rejeitos sólidos & 0,25 & - & 4,2 & 1,2 & 2,6 & 0,26 \\
\hline Escoamento urbano & 5,2 & 0,77 & 0,04 & 0,008 & 0,01 & 0,53 \\
\hline Total - Rio Macaé & 1.315 & 647 & 24 & 6,0 & 7,6 & 1,9 \\
\hline Estuários $(\mathrm{CE})^{2}$ & 1.645 & 622 & - & - & - & - \\
\hline Rio de Contas (BA) ${ }^{21}$ & 398 & 241 & 18 & 6,4 & 5,6 & 3,5 \\
\hline Estuários (RN) ${ }^{22}$ & 5.080 & 1.590 & - & - & - & - \\
\hline Baía de Sepetiba (RJ) ${ }^{75}$ & - & - & 6.750 & - & 386 & 34,8 \\
\hline
\end{tabular}

expressas através de distribuição de probabilidades, identificando parâmetros e categorias de fontes de emissão que possuem a maior contribuição para a incerteza global do inventário, com o objetivo de prover informações que possam ser aperfeiçoadas posteriormente. ${ }^{76}$

Considerando a reduzida disponibilidade e abrangência de dados para alguns parâmetros das estimativas do presente estudo, a incerteza foi avaliada através do cálculo da faixa de variação das emissões por deposição atmosférica e por águas servidas que apresentaram mais abrangência de dados. A faixa de variação das emissões via deposição atmosférica $\left(\mathrm{t} \mathrm{ano}^{-1}\right)$ foi $\mathrm{N}$ : 26,6-366; P: 0,20-4,6; Zn: 0,60-6,5; $\mathrm{Cu}$ : 0,21-1,9; Pb: 1,1-8,1; Cd: 0,22-3,1. Para as emissões por águas servidas, as faixas de variação observadas foram $\mathrm{Zn}$ : 0,98-7,3; $\mathrm{Cu}$ : 1,0-2,9; Pb: 0,22-1,5; Cd: 0,015-0,22. As variações reportadas entre os valores mínimos e máximos de ambos os fatores estão dentro das faixas de variação das emissões propostas na literatura, ${ }^{20}$ indicando que os fatores de emissão calculados no presente estudo podem variar de 4 a 23 vezes, até uma ordem de grandeza, sendo esta a variabilidade extrapolada dependendo do elemento químico e da categoria de emissão representada no presente estudo.

As menores incertezas estão associadas à deposição atmosférica, águas servidas e escoamento urbano, pois apresentam na literatura uma abrangência razoável de dados regionais sobre as concentrações e fluxos de nutrientes e metais para estas fontes. As maiores incertezas estão associadas à emissão por resíduos sólidos, devido à diversidade de resíduos e concentrações associadas, assim como a distribuição/ retenção pela diversidade de solos que também pode explicar as maiores incertezas da denudação dos solos, agricultura e pecuária. Deve-se considerar que a precisão requerida pelos fatores de emissão depende da expectativa dos usos dos dados gerados que, no caso do presente estudo, visa uma primeira aproximação das emissões, sendo necessários estudos futuros visando diminuir as incertezas e aproximar os resultados à realidade da área de estudo.

Adicionalmente, a exatidão dos cálculos dos fatores de emissão foi obtida através da comparação com as medições dos fluxos de N, $\mathrm{P}, \mathrm{Cu}, \mathrm{Pb}, \mathrm{Cd}$ e $\mathrm{Zn}$ transportados pelo Rio Macaé em Novembro de 2010 na porção final da bacia sob influência urbana. Os resultados medidos sugerem fluxos anuais de $\mathrm{N}$ : $1106 \mathrm{t} \mathrm{ano}^{-1}$; $\mathrm{P}: 362 \mathrm{t} \mathrm{ano}^{-1}$; $\mathrm{Zn}$ : $6,5 \mathrm{t} \mathrm{ano}^{-1} ; \mathrm{Cu}: 2,3 \mathrm{t} \mathrm{ano}^{-1} \mathrm{e} \mathrm{Pb}: 4,3 \mathrm{t} \mathrm{ano}^{-1}$. Os valores de Cd ficaram abaixo do limite de detecção do método analítico, não permitindo o cálculo dos fluxos.

Os fluxos foram medidos a partir de concentrações que se apresentaram inferiores a ambientes fluviais com histórico de contaminação como o Rio Paraíba do Sul ${ }^{77}$ e a Baía de Sepetiba, ${ }^{78}$ como no caso do $\mathrm{N}$ e $\mathrm{Zn}$, embora para $\mathrm{Cu}$ e $\mathrm{Pb}$ as concentrações medidas fossem superiores, podendo ser explicadas pelo caráter urbano da porção final da bacia que recebe efluentes sem tratamento (Tabela 7).

Comparando as razões entre os valores estimados e medidos pode-se observar similaridade bastante razoável para $\mathrm{N}(1,4)$ e $\mathrm{Pb}$ $(1,8)$ e mais discrepante para $\mathrm{P}(2,1)$. Para $\mathrm{Zn}(4,5)$ e $\mathrm{Cu}(3,0)$ os valores estimados foram superiores aos valores medidos. Os resultados são bastante consistentes, entretanto, uma vez que a medida instantânea foi realizada em um período hidrológico intermediário, esta não incluiu as maiores cargas, particularmente de metais, que são tipicamente transportadas em períodos de pluviosidade intensa. Por outro lado, as concentrações de metais nos substratos e efluentes considerados são bem mais variáveis e específicas que os nutrientes. Portanto, para melhor estimar suas cargas seriam necessárias medidas de suas concentrações nos substratos gerados localmente, ao invés de dados da literatura. Entretanto, mesmo considerando as maiores diferenças $(\mathrm{Zn} \mathrm{e} \mathrm{Cu})$ as estimativas de carga mostraram-se bastante consistentes e capazes de gerar cenários factíveis à tomada de decisão quanto ao controle de suas fontes.

Tabela 7. Concentração/desvio padrão: faixa de variação (fluxo) de N, Zn, Cu e Pb no material particulado em suspensão nas bacias do Rio Macaé, Rio Paraíba do $\mathrm{Sul}^{42,77}$ e Baía de Sepetiba ${ }^{78}\left(\mathrm{~N}: \mathrm{mg} \mathrm{L}^{-1}\right.$; metais: $\mu \mathrm{g} \mathrm{g}^{-1}$; fluxos: $\left.\mathrm{t} \mathrm{ano}^{-1}\right)$

\begin{tabular}{|c|c|c|c|c|}
\hline & $\mathrm{N}$ & $\mathrm{Zn}$ & $\mathrm{Cu}$ & $\mathrm{Pb}$ \\
\hline Macaé & $\begin{array}{c}0,51 / 0,30: 0,38-0,96 \\
(1.354)\end{array}$ & $\begin{array}{c}\text { 192/136: } 51-350 \\
(6,5)\end{array}$ & $\begin{array}{c}90 / 33: 41-113 \\
(2,3)\end{array}$ & $\begin{array}{c}\text { 170/129: } 24-297 \\
(4,3)\end{array}$ \\
\hline Baía de Sepetiba & - & $\begin{array}{c}\text { 412: } 219-798 \\
\text { (12) }\end{array}$ & $\begin{array}{c}76: 26-116 \\
(2,7)\end{array}$ & $\begin{array}{c}\text { 116: } 51-320 \\
(4,5)\end{array}$ \\
\hline Rio Paraíba do Sul & $\begin{array}{c}0,77 \\
(26.000)\end{array}$ & $\begin{array}{c}\text { 297/178: } 34-787 \\
(345)\end{array}$ & $\begin{array}{c}\text { 75/41: } 60-202 \\
(214)\end{array}$ & - \\
\hline
\end{tabular}




\section{CONCLUSÃO}

Os resultados das cargas estimadas através de fatores de emissão indicam que as fontes antrópicas são responsáveis pela maior parte das emissões de nutrientes e metais para o Rio Macaé, sendo muito maiores que as emissões naturais para nutrientes e em menor escala para $\mathrm{Zn}$ e $\mathrm{Cu}$, e equivalentes para $\mathrm{Pb}$ e $\mathrm{Cd}$. A pecuária e a liberação de águas servidas (esgoto doméstico) são os principais responsáveis pelo incremento antrópico da emissão dos elementos químicos, enquanto que para $\mathrm{Pb}$, a disposição de rejeitos sólidos é a fonte principal. Desta forma, ao lado da pecuária que é vocação regional da bacia do Rio Macaé, observa-se que a urbanização e o crescimento socioeconômico estimulado pelas atividades de exploração e petróleo na Bacia de Campos induzem ao aumento das emissões de elementos químicos para o Rio Macaé.

\section{AGRADECIMENTOS}

Este trabalho foi financiado pela FAPERJ (proc. 110.956.2008) e CNPq (476497/2010-8). Os resultados deste estudo fazem parte do âmbito do projeto Instituto Nacional de Ciência e Tecnologia de Transferência de Materiais Continente-Oceano (Proc. No. 573.601/2008-9).

\section{REFERÊNCIAS}

1. Bricker, S. B.; Clement, C. G.; Pirhalla, D. E.; Orlansdo, S. P.; Farrow, D. R. G.; National Estuarine Eutrophication Assessment: effects of Nutrient Enrichment in the Nation's Estuaries, NOAA: Silver Spring, 1999.

2. Lacerda, L. D.; Molisani, M. M.; Senna, D.; Maia, L. P.; Environ. Monit. Assess. 2008, 141, 149.

3. Lacerda, L. D.; Molisani, M. M.; Mar. Pollut. Bull. 2006, 52, 969.

4. Machado, W.; Moscatelli, M.; Rezende, L. G.; Lacerda L. D.; Environ. Pollut. 2002, 120, 455.

5. Oygard, J. K.; Mage, A.; Gjengedal, E.; Water Res. 2004, 38, 2851.

6. Silva Filho, E. V.; Sella, S. M.; Spinola, E. C.; Santos, I. R.; Machado, W.; Lacerda, L. D.; Microchem. J. 2006, 82, 196.

7. Davis, A. P.; Shokouhian, M.; Shubei, M.; Chemosphere 2001, 44, 997.

8. Sorme, L.; Lagerkvist, R.; Sci. Total Environ. 2002, 298, 131.

9. Prestes, E. C.; Anjos, V. E.; Sodré, F. F.; Grassi, M. T.; J. Braz. Chem. Soc. 2006, 17, 53.

10. Lima, J. S.; Lichtig, J.; Oliveria, E.; Menk, J. R. F.; Rev. Ceres 1999, 46, 571.

11. Gimeno-García, E.; Andreu, V.; Boluda, R.; Environ. Pollut. 1996, 92, 19.

12. Mortvedt, J. J.; Fertilizer Res. 1996, 43, 55.

13. Ramalho, J. F. G. P.; Amaral Sobrinho, N. M. B.; Flor. Amb. 2001, 8, 120.

14. Ramalho, J. F. G. P.; Amaral Sobrinho, N. M. B.; Velloso, A. C. X.; Pesq. Agropec. Bras. 2000, 35, 1289.

15. Paiva, D. P.; Anais do Seminário Internacional de Aves e Suínos, Florianópolis, Brasil, 2005.

16. Botello, A.V.S.; Villanueva, F.; Diaz, G.; Rev. Environ. Contam. Toxicol. 1997, 153, 91.

17. Henderson, S. B.; Grigson, S. J. W.; Johnson, P.; Roddie, B. D.; Mar. Pollut. Bull. 1999, 38, 1141.

18. Pinheiro, M. R. C.; Dissertação de Mestrado, Centro Federal de Educação Tecnológica de Campos, Brasil, 2008.

19. http://www.ibge.gov.br/cidadesat/topwindow.htm?1, acessada em Novembro 2012.

20. Nriagu, J. O.; Pacyna, J. M.; Nature 1988, 333, 134.

21. de Paula, F. C. F.; Lacerda, L. D.; Marins, R. V.; Aguiar, J. E.; Ovalle, A. R. C.; Falcão Filho, C. A. T.; Quim. Nova 2010, 33,70.
22. Lacerda, L. D.; Vaisman, A. G.; Maia, L. P.; Silva, C. A. R.; Cunha, E. M. S.; Aquaculture 2006, 253, 433.

23. Lacerda, L. D.; Marins, R. V.; J. Geochem. Explor. 1997, 58, 223.

24. Lacerda, L. D.; Santos, J. A.; Madrid, R. M.; Mar. Pollut. Bull. 2006, 52, 1823.

25. Lacerda, L. D.; Santos, A. F.; Marins, R. V.; Quim. Nova 2007, 30, 366.

26. Bouwman, A. F.; Lee, D. S.; Asman, A. H.; Dentener, F. J.; van der Hoek, K. W. A.; Olivier, J. G. J.; Global Biogeochem. Cycles 1997, 11, 561.

27. Lacerda, L. D.; Environ. Geol. 2003, 43, 308.

28. NRC; Clean Coastal Waters. Understanding and Reducing the Effects of Nutrient Pollution, National Research Council, Academy Press: Washington, 2003.

29. Malavolta, E.; Dantas, J. P. Em Melhoramento e Produção do Milho no Brasil; Paterniani, E., ed.; Fundação Cargill: São Paulo, 1980, cap. 13.

30. Ferreira. J. M. S.; Produção Integrada de Coco, EMBRAPA Tabuleiros Costeiros: Aracaju, 2003

31. Silva, E. C.; Silva Filho, A. V.; Almeida, M. A. R.; Ciênc. Agrotecnol. 2000, 24, 509.

32. Lima, J. S. L.; Agr. Ecosyst. Environ. 1994, 48, 19.

33. Tundisi, J. G.; Água no Século XXI: Enfrentando a Escassez, Rima: São Carlos, 2006.

34. Lacerda, L. D.; Braz. J. Aquat. Sci. Technol. 2006, 10, 13.

35. ABES; Catálogo Brasileiro de Engenharia Sanitária, Associação Brasileira de Engenharia Sanitária e Ambiental: Rio de Janeiro, 1983.

36. von Sperling, M.; Introdução à Qualidade das Águas e ao Tratamento de Esgotos, Universidade Federal de Minas Gerais: Belo Horizonte, 1996.

37. Mello, W. Z.; Environ. Pollut. 2001, 114, 235.

38. Mello, W. Z.; Almeida, M. D.; Environ. Pollut. 2004, 129, 63.

39. Marins R. V.; Silva Filho, E. V.; Lacerda, L. D.; J. Braz. Chem. Soc. 1996, 7, 177.

40. Tan, P. A.; Wong, A. Y. S.; Water, Air, Soil Pollut. 2000, 122, 261.

41. Valderrama, J. C.; Mar. Chem. 1981, 10, 109.

42. Salomão, M. S. M. B.; Molisani, M. M.; Ovalle, A. R. C.; Rezende, C. E.; Lacerda, L. D.; Carvalho, C. E. V.; Hydrol. Process. 2001, 15, 587.

43. Schlesinger, W. H.; Biogeochemistry: An analysis of global change, Academic: San Diego, 1997.

44. Goudie, A.; The Human Impact: on the Natural Environment, MIT Press: Cambridge, 1987.

45. Greenland, D. J.; Lal, R.; Soil conservation and management in the humid tropics, Wiley: Chischester, 1977.

46. Inácio, E. S. B.; Cantalice, J. R. B.; Nacif, P. G. S.; Araújo, Q. R.; Barreto, A. C. R.; Rev. Bras. Eng. Agríc. Ambient. 2007, 11, 355.

47. Dias, C. M. F.; Souza, C. M. M.; Moneratti, P. H.; Anais do X Congresso Brasileiro de Geoquímica, Curitiba, Brasil, 2001.

48. Holmgren, G. G. S.; Meyer, M. W.; Chaney, R. L.; Daniels, R. B.; J. Environ. Qual. 1993, 22, 335.

49. Silva, L. F.; Solos Tropicais: Aspectos Pedológicos, Ecológicos e de Manejo, Terra Brasilis: São Paulo, 1996.

50. Canellas, L. P.; Velloso, A. C. X.; Marciano, C. R.; Ramalho, J. F. G. P.; Rumjanek, V. M.; Rezende, C. E.; Santos, G. A.; R. Bras. Ci. Solo 2003, 27, 935.

51. Mesquita, E. E.; Pinto, J. C.; Furtini Neto, A. E.; Santos, I. P. A.; Tavares, V. B.; R. Bras. Zootec. 2004, 33, 290.

52. Andrade, C. A.; Mattiazzo, M. E.; Scien. Florest. 2000, 58, 59.

53. Fadigas, F. S.; Amaral Sobrinho, N. M. B.; Mazur, N.; Anjos, L. H. C.; Freixo, A. A.; Rev. Bras. Eng. Agríc. Ambient. 2006, 10, 699.

54. Brunner, U.; Bachofen, R.; Toxicol. Environ. Chem. 1998, 67, 171.

55. Embrapa Solos, Ministério da Agricultura, Folha SF-24-Y-A Macaé, 2003.

56. Burns, D. A.; Environ. Pollut. 2004, 127, 257.

57. Golley, F. B.; Mc Guiness, J. T.; Clements, R. G.; Child, G. I.; Duever, M. J.; Ciclagem de Minerais em um Ecossistema de Floresta Tropical Úmida, EDUSP: São Paulo, 1978. 
58. Johnson, D. W.; Lindberg, S. E.; Atmospheric Deposition and Forest Nutrient Cycling, Springer Verlag: Berlin, 1998.

59. Lindberg, S.; Harris, R. C.; Water, Air, Soil Pollut. 1981, 16, 13.

60. Schlesinger, W. H.; Gray, J. T.; Gilliam, F. S.; Wat. Resour. Res. 1982, 18,623 .

61. Silva Filho, E. V.; Wasserman, J. C.; Lacerda, L. D.; Ciência e Cultura 1998, 50, 374 .

62. http://www.agrobyte.com.br/cana.htm, acessada em Novembro 2012.

63. http://www.embrapa.br, acessada em Novembro 2012.

64. Bouwman, A. F.; Lee, D. S.; Asman, A. H.; Dentener, F. J.; van der Hoek, K. W. A.; Olivier, J. G. J.; Global Biogeochem. Cycles 1997, 11, 561.

65. Vollenweider, R. A.; Scientific Fundamentals of the Eutrophication of Lakes and Flowing Waters with Particular Reference to Nitrogen and Phosphorus as Factors in Eutrophication, OECD: Paris, 1968.

66. Howarth, R. W.; Billen, D.; Swaney, A.; Townsend, N.; Janarski, K.; Lajtha, K.; Downing, J. A.; Elmgren, R.; Caraco, N.; Jordan, T.; Berendse, F.; Freney, J.; Kudeyorov, V.; Murdcoh, P.; Zhao-Liang, Z.; Biogeochemistry 1996, 46, 203.

67. Boyd, C. E.; Am. Fish. Soc. Spec. Publ. 1971, 8, 153.

68. Menzi, H.; Kessler, J.; Proceedings of the $8^{\text {th }}$ International Conference of the FAO Network on Recycling of Agricultural. Municipal and Industrial Residues in Agriculture, Rome, Itália, 1998.
69. Ambrus, A.; Hamilton, D. J.; Kuiper, H. A.; Racke, K. D.; Pure Appl. Chem. 2003, 75, 937.

70. Bouwman, A. F.; Booij, H.; Nutr. Cycl. Agroecosyst. 1998, 52, 262.

71. Smith, R. A.; Alexander, R. B.; Wolman, M. G.; Water Resour. Res. 1997, 33, 2781.

72. C. Consultants Ltd.; Pollutants in Urban Waste Water and Sewage Sludge, The Office for Official Publications of the European Communities: Luxembourg, 2001.

73. Doll, P.; Hauschild, M.; Reg. Environ. Change 2002, 2, 150.

74. Binner, E.; Lechner, P.; Ziegler, C.; Riehl-Herwirsch, G.; Breitenau Landfill - Water Balance, Emissions and a Look into the Landfill Body, Breitenau Landfill Co.: Viena, 1996.

75. Molisani, M. M.; Marins, R. V.; Machado, W. T.; Paraquetti, H. H. M.; Bidone, E. D.; Lacerda, L. D.; Reg. Environ. Change 2004, 4, 17.

76. USEPA; Emission factor uncertainty assessment. Review Draft. 2007.

77. Figueiredo, R. O.; Ovalle, A. R. C.; Rezende, C. E.; Martinelli, L. A.; Rev. Ambi-Água, 2011, 6, 7.

78. Lacerda, L. D.; Pfeiffer, W. C.; Fiszman, M.; Sci. Total Environ. 1987, $65,163$. 